\title{
EVOLUCIÓN DE LA MERITOCRACIA EN EL SERVICIO CIVIL EN ECUADOR.
}

\section{EVOLUTION OF MERITOCRACY IN CIVIL SERVICE IN ECUADOR.}

\author{
Irma Victoria Jara Íñiguez, PhD. \\ Doctora en Administración (Ecuador). \\ Docente en el Instituto de Altos Estudios Nacionales (IAEN). \\ Gerente General del Centro de Educación Continua del IAEN, Ecuador. \\ ijarainiguez@gmail.com
}

\section{ARTÍCULO DE REFLEXIÓN}

Recibido: 5 de enero de 2017.

Aceptado: 27 de febrero de 2017.

\section{RESUMEN}

El presente es un estudio sobre la evolución de la meritocracia en Ecuador en el periodo 2006-2016, en el que se analiza el grado de avance y cumplimiento de los principales elementos de un sistema meritocrático, respecto al ingreso al servicio civil y la carrera en la Función Ejecutiva. Se identifican los hitos en cuanto a la normativa e instrumentos desarrollados, acciones implementadas y se contrasta con los resultados obtenidos en mediciones e informes internacionales, conforme a la Carta Iberoamericana de la Función Pública y la Convención Interamericana contra la Corrupción. Se utilizó un análisis cualitativo y documental, con un esquema basado en la comparación diacrónica en dos fases, teniendo en cuenta como aspecto relevante, los cambios legales y éstos como contexto para las acciones públicas y los cambios institucionales realizados. Sobre la base de un análisis y valoración en forma binaria, se identificaron -a partir del 2014avances y mejoras respecto a la selección de servidores, en cuanto a: concursos de méritos y oposición de modalidad abierta, aplicación de pruebas, publicación y transparencia de procesos, fiabilidad de las herramientas y equidad. Respecto a la evaluación de desempeño, sin embargo, se evidenciaron debilidades a lo largo del periodo analizado.

Palabras clave: administración pública, carrera administrativa, Ecuador, mérito, servicio público. 


\section{ABSTRACT}

The present is a study on the evolution of meritocracy in Ecuador in the period 2006-2016, in which the degree of progress and fulfillment of the main elements of a meritocratic system, regarding the entrance to the civil service and the administrative career in the Executive Function is analized. It identifies events in terms of regulations and instruments developed, actions implemented and contrasts with the results obtained in international measurements and reports, according to the Ibero-American Charter of Public Function and the Inter-American Convention against Corruption. It was used a qualitative and documentary analysis, with a scheme based on the diachronic comparison in two phases, taking into account as relevant aspect, legal changes and these as a context for public actions and institutional changes. On the basis of binary analysis and assessment, as of 2014, progress was made on the selection of civil servants in terms of merit and open contests, application of tests, publication and transparency process, tool reliability and equity. Regarding the performance evaluation, however, weaknesses were evidenced throughout the analyzed period.

Keywords: public administration, administrative career, Ecuador, merit, public service.

\section{INTRODUCCIÓN}

El Estado, a través de sus instituciones, está llamado a proveer a la ciudadanía de servicios públicos de calidad, para hacer efectivo el buen vivir, por ello la formulación, ejecución, evaluación y control de las políticas públicas y servicios deben garantizar el ejercicio de los derechos reconocidos por la Constitución, siendo esencial asegurar para su cumplimiento una gestión pública eficiente.

La administración y la política están interrelacionados ya que la primera interactúa en las fases de construcción de políticas públicas, fundamentalmente en la fase de implementación influyendo por tanto en los resultados del sistema político, a su vez, es crucial para el desarrollo del componente objetivo subjetivo de la política pública, ya que los servidores entran en contacto con la ciudadanía, y por tanto personifican las acciones del estado a través de su desempeño. (Jara, 2015)

Una administración que funciona bajo parámetros de un servicio civil basado en el mérito en el ingreso y carrera, que no permita la incidencia de prácticas clientelares, a través de su vínculo con la capacidad estatal para implementar políticas viabiliza la implementación de los objetivos de gobierno y, la entrega de servicios de calidad. 
Ecuador ha implementado varias acciones en la administración pública con el objetivo de lograr este cometido, por lo que, el estudio está dirigido a realizar un análisis diacrónico comparado por fases para evidenciar cómo el País ha evolucionado en los últimos 11 años respecto a institucionalidad, prácticas y resultados en el ámbito de la meritocracia, en específico a los procesos de selección y evaluación de los servidores; así como, el grado de coherencia entre la normativa e instrumentos desarrollados y las acciones implementadas desde las instituciones del Estado, y contrastando con los resultados obtenidos en mediciones internacionales.

El análisis se enmarca en el servicio civil ecuatoriano, enfocado en la administración pública central, institucional y dependiente de la Función Ejecutiva para el periodo 20062016, con dos fases con marco legal diferenciado 2006-2010 y 2011-2016. El estudio identifica las políticas e instrumentos aplicados para el ingreso al servicio público y la carrera administrativa, los avances de las acciones relacionadas con la meritocracia y, sus resultados conforme el índice de desarrollo del servicio civil del BID en el marco de los principios de la Carta Ibeoramericana de la Función Pública y, las recomendaciones del mecanismo de Seguimiento de la Implementación de la Convención Interamericana contra la Corrupción.

Se concluye como, dentro del cumplimiento del accionar del Estado, los cambios en la institucionalidad en el servicio público respecto al sistema meritocrático favorecen los resultados del índice de desarrollo servicio civil y el enfoque de transparencia y lucha contra la corrupción. Al 2016, se destacan diversos avances en cuanto al sistema de ingreso al servicio público por concursos de méritos y oposición abiertos y una mejora en la medición del índice de desarrollo de servicio civil con un aumento en 26 puntos, el de mérito con aumento en 33 puntos y, de gestión del empleo, 30 puntos.

\section{REVISIÓN TEÓRICA}

\section{Marco Conceptual y Legal.}

Acorde con la definición de la enciclopedia de la política de Rodrigo Borja (2012) la meritocracia responde al principio de la igualdad de oportunidades en la vida social y a la eliminación de los privilegios provenientes de varios factores discriminatorios, y su origen está en la Declaración de los Derechos del Hombre y del Ciudadano (1789) que estableció que las personas son igualmente admisibles a todas las dignidades, puestos y empleos públicos, según su capacidad y sin otra distinción que aquella de su virtud y de su ingenio. Con este marco, inicial y, acogiendo la definición de la Real Academia 
Española (2014) a la palabra meritocracia, como un "sistema de gobierno en que los puestos de responsabilidad se adjudican en función de los méritos personales", se analizará la evolución de la meritocracia, sus sistemas, instrumentos e implementación en Ecuador en la década 2006-2016.

El análisis de las políticas públicas de gestión de talento humano y, en específico las acciones en el marco del sistema de meritocracia en el servicio público, es trascendental para la sociedad, porque como indican lacoviello y Chudnovsky (2015) el rol del aparato administrativo es central en la elaboración y la implementación de las políticas públicas y en la relación que se establece entre el Estado y los ciudadanos; por lo que, la forma en que en la práctica se seleccionan los servidores en las instituciones públicas es fundamental e incidente en los resultados de las políticas en beneficio de la sociedad.

La administración y la política no son fenómenos discretos sino que están interrelacionados, y como los servidores entran en contacto con los agentes, actores y usuarios, en la fase final las acciones del Estado se personifican en ellos y su desempeño La eficacia y eficiencia de la acción de gobierno, se alcanza si los servidores cumplen con los requerimientos de profesionalidad y capacidad, los cuales son propios de un sistema de mérito según indica el BID (2001) en lacoviello y Chudnovsky (2015). Para que los ciudadanos, en una sociedad democrática, gocen de una buena administración pública se debe proveer de un sistema de empleo público meritocrático, transparente, flexible y eficiente (lacoviello y Chudnovsky, 2015) .

La Carta Iberoamericana, respaldada por la XIII Cumbre Iberoamericana de Jefes de Estado y de Gobierno en Bolivia en noviembre de 2003 (Resolución № 11 de la "Declaración de Santa Cruz de la Sierra") de la cual es signatario Ecuador, entre los principios rectores de las políticas de gestión de empleo y recursos humanos en la función pública prescribe al mérito, desempeño y capacidad como criterios orientadores del acceso a la carrera (así como para directivos) y las restantes políticas de recursos humanos; se recomienda que se introduzcan regulaciones y especificidades para garantizar que la gestión del empleo y las personas que lo integran estén protegidas de politización, clientelismo, arbitrariedad y captura por intereses particulares y, así mismo articulación para el cambio cultural en ese sentido.

A partir de la Carta Iberoamericana (2003), el BID desarrolla una medición de los servicios civiles alineada a dichos principios, utilizando una metodología con puntos críticos, atendiendo cinco índices de calidad (eficiencia, mérito, capacidad funcional, consistencia estructural, capacidad integradora) y 8 subsistemas de la gestión de recursos humanos 
(planificación de recursos humanos, organización del trabajo, gestión del empleo, gestión del rendimiento, gestión de la compensación, gestión del desarrollo, gestión de las relaciones humanas y sociales, y organización de la función de recursos humanos); esta medición se realizó la primera vez en el 2004, para 16 países de América Latina. Posteriormente, se aplicó a través de la metodología calibrada de Longo (2006) para simplificar y favorecer la comparabilidad.

En esta medición, respecto al subsistema de gestión del empleo se plantean varios elementos como fundamentales para un sistema meritocrático: concurso de mérito como mecanismo de ingreso; concursos abiertos y de amplia difusión; concursos basados en pruebas técnicas de selección (que incluyan competencias). Se debe contemplar publicidad, transparencia en la gestión de los procesos, con idoneidad de los cuerpos técnicos encargados de la selección, además de imparcialidad y de la fiabilidad de las herramientas que se instrumentan para la selección. En cuanto a la gestión de rendimiento se destacan como instrumentos complementarios recomendables: evaluación institucional; evaluación para altos funcionarios públicos; evaluación individual; modelo de gestión estratégica de evaluación de desempeño. (BID, 2014)

En la metodología, el índice de Mérito mide las garantías de profesionalismo en el funcionamiento del sistema de servicio civil y valora el grado de imparcialidad de las decisiones dentro de cada subsistema de gestión. Mide, en concordancia con lo estipulado en la Carta Iberoamericana el grado de protección efectiva que el sistema ofrece frente a la arbitrariedad, la captura política o clientelista y las diferentes modalidades de búsqueda de rentas por parte de grupos o sectores interesados. (BID, 2014)

La convención Interamericana contra la corrupción, adoptada en Caracas en 1996 y en vigor desde 1997, de la cual Ecuador es signatario, indica entre sus propósitos el de promover, facilitar y regular la cooperación entre los Estados Partes a fin de asegurar la eficacia de las medidas y acciones para prevenir, detectar, sancionar y erradicar los actos de corrupción en el ejercicio de las funciones públicas y los actos de corrupción específicamente vinculados con tal ejercicio. Entre las medidas preventivas de la mencionada convención, se cuenta la relacionada con los sistemas para la contratación de funcionarios públicos por parte del Estado que aseguren la publicidad, equidad y eficiencia de tales sistemas. (OEA, 1996)

El Mecanismo de Seguimiento de la Implementación de la Convención Interamericana (OEA)2001, es un instrumento de carácter intergubernamental establecido en el marco 
de la OEA para apoyar a los Estados -que son parte del mismo- en la implementación de las disposiciones de la Convención, mediante un proceso de evaluaciones recíprocas y en condiciones de igualdad, en donde se formulan recomendaciones específicas con relación a las áreas en que existan vacíos o requieran mayores avances. El mecanismo no sanciona, ni califica o clasifica a los Estados sino que fortalece la cooperación entre éstos. (OEA, 2011)

EI MESICIC está conformado por la Conferencia de los Estados Parte, con la autoridad y responsabilidad de instrumentar el mecanismo, el Comité de Expertos, responsable del análisis técnico y la Secretaría Técnica ejercida por la Secretaría General de la OEA, a través del departamento de Cooperación Jurídica. (OEA, 2011)

Las evaluaciones se realizan en rondas sucesivas en las cuales se verifican los avances respecto a la convención, como resultado se adoptan informes nacionales en los que se formulan recomendaciones concretas para que llenen los vacíos normativos detectados; subsanen las inadecuaciones encontradas; y cuenten con indicadores que permitan determinar objetivamente los resultados obtenidos con relación a la implementación de tales disposiciones, cabe indicar que las organizaciones de la sociedad civil participan en este proceso proveyendo información; al terminar una ronda, el Comité adopta un Informe Hemisférico. (OEA, 2011)

\section{Breve contexto.}

Al 2006 se encontraba vigente la Constitución Política de la República del Ecuador de 1998, que prescribía en su artículo 124 que la administración pública se organizará de manera descentralizada y desconcentrada y la Ley garantizará los derechos y establecerá las obligaciones de los servidores públicos, regulará su ingreso, estabilidad, evaluación, ascenso y cesación. El ingreso y ascenso dentro del servicio civil y la carrera administrativa se realizarán mediante concursos de mérito y oposición, y sólo por excepción estarán sujetos a un régimen de libre nombramiento y remoción.

Para el régimen laboral del servicio civil, en general, existía una diversidad de regulaciones, podían estar amparados por la Ley Orgánica del Servicio Civil y Carrera Administrativa, Homologación y Unificación de las remuneraciones del sector público LOSCCA- codificada al 2005 (R.O 16 de 12 de Mayo de 2005), y otras leyes o regímenes especiales, según el caso. A la LOSCCA se realizaron varias reformas hasta su derogación en el 2010 con la Ley Orgánica del Servicio Público, LOSEP.

La Constitución promulgada en el 2008 en sus artículos 227 y 228 indica que la 
administración pública constituye un servicio a la colectividad que se rige entre otros por los principios de eficacia, eficiencia, calidad, transparencia y evaluación; y el ingreso al servicio público, el ascenso y la promoción en la carrera administrativa se realizarán mediante concurso de méritos y oposición, con excepción de las servidoras y servidores públicos de elección popular o de libre nombramiento y remoción, con la sanción caso de inobservancia de destitución de la autoridad nominadora. En este, marco se expidió la LOSEP desde su promulgación en octubre de 2010 (R.O. Suplemento 294 de 6 de Octubre de 2010), hasta diciembre de 2016 ha recibido 7 reformas de distintos alcances, algunos relacionados con el ingreso, carrera y evaluación de los servidores.

Respecto del MESICIC, en Ecuador se han desarrollado cuatro rondas en el periodo de análisis, desde la II a la V ronda, siendo el informe de esta última aprobado en septiembre de 2016, indicándose que en cuanto a los sistemas de contratación (vinculación) de los servidores públicos -en el ámbito de la administración central, institucional y dependiente de la Función Ejecutiva- se destacan diversos avances, especialmente la aprobación de la Ley Orgánica de Servicio Público (R.O. 294, 6 de Octubre de 2010), y su Reglamento (R.O. 416, 1 de abril de 2011).

\section{MATERIALES Y MÉTODOS}

Se utilizó para el estudio un análisis cualitativo, a partir de una investigación documental del 2006-2016. Se analizó también la información de carácter cuantitativo, buscando generar datos descriptivos, privilegiando el acceso al significado de las acciones desde la perspectiva y percepción de expertos, organismos internacionales (como seguimiento de segunda parte) y la opinión de la ciudadanía a través de lo expresado por organizaciones sociales.

Para la selección de la documentación se tuvo en consideración criterios académico, institucional, internacional, y de la ciudadanía. Se trabajó con un esquema basado en un estudio diacrónico del marco legal, institucional, herramientas utilizadas, prácticas e informes internacionales, de las políticas de gestión de recursos humanos en el servicio público en el país a lo largo de 11 años.

Para la determinación de aspectos de relevancia en la identificación de las fases del presente análisis, se consideraron hitos en dichos elementos, en relación a la aplicación de las políticas públicas de Gestión de Talento Humano en Ecuador, entre ellos: vigencia de las Constituciones Políticas, vigencia de Leyes en torno al servicio civil, reformas normativas y cambios institucionales. 
Se identifican dos fases relevantes la fase 1 de 2006-2010, en la que se identifica un marco constitucional con orientación empresarial y a partir de octubre de 2008 una constitución con orientación centrada en el Estado, cambio de legislación al final del periodo, cambio en la rectoría y la fase 2 de 2011-2016, con un solo marco constitucional y una ley con varias reformas -solo una con énfasis para el efecto de este estudio-, ajustes al ente rector -cambio de denominación-.

Para medición de la evolución y avances de la meritocracia en el servicio civil se tomó como principal referencia -a efectos académicos y sustantivos-: a) el índice de desarrollo de servicio civil, los índices de calidad del servicio civil, en específico el índice de mérito, y la valoración -puntajes- de los subsistemas de gestión del empleo y gestión del rendimiento que son publicados por Banco Interamericano de Desarrollo, desde 2004, y que mide, los niveles de desarrollo que van desde alto (60-100 puntos), Medio (40-59 puntos) a bajo (hasta 39 puntos), los niveles son el resultante de la evaluación del desempeño del sistema del servicio civil en función de los principios de la Carta Iberoamericana de la Función Pública. b) Los informes finales de País de la MESICIC, aprobados en plenaria, respecto a la identificación del cumplimiento de los compromisos en la lucha contra la corrupción, acorde a lo establecido en la convención interamericana contra la corrupción, respecto al ámbito de contratación de servidores públicos, y en aspectos de transparencia y cobertura con que se gestiona los concursos de méritos y oposición.

A partir de estas referencias, la base del análisis documental y, adicionalmente cuando fue aplicable a través de la verificación, se identificó el cumplimiento de elementos de un sistema meritocrático y se realizó una valoración en forma binaria (0-1: verdadero-falso), sobre las acciones ejecutadas por fases en el periodo establecido.

\section{RESULTADOS Y DISCUSIÓN}

\section{Institucionalidad y características del sistema meritocrático.}

En las siguientes tablas se describen las características de la institucionalidad y el sistema, sobre la base de lo estipulado en la Legislación vigente, por fases. 
Tabla 1 A. Institucionalidad y características de ingreso. Fase 1.

\begin{tabular}{|c|c|c|c|c|c|}
\hline Institucionalidad & 2006 & 2007 & 2008 & 2009 & 2010 \\
\hline Constitución & \multicolumn{3}{|c|}{ Constitución 1998; Constitución 2008} & \multicolumn{2}{|c|}{ Constitución 2008} \\
\hline Ley & \multicolumn{5}{|c|}{$\begin{array}{l}\text { Ley Orgánica de Servicio Civil y Carrera Administrativa y de Unificación y } \\
\text { Homologación de las Remuneraciones del Sector Público(Registro Oficial } \\
16 \text { de } 12 \text { de Mayo de 2005), y sus reformas } 2006,2007,2008,2009 . \\
\text { Reglamento a la LOSCCA (R.O Suplemento } 505 \text { de } 17 \text { de Enero de } \\
\text { 2005) y reformas. } \\
\text { Ley Orgánica del Servicio Público. Registro Oficial Suplemento } 294 \text { de } 6 \\
\text { de Octubre de } 2010\end{array}$} \\
\hline Rectoría & \multicolumn{4}{|c|}{$\begin{array}{l}\text { Secretaría Nacional Técnica de Desarrollo de Recursos } \\
\text { Humanos y Remuneraciones del Sector Público, SENRES }\end{array}$} & $\begin{array}{l}\text { Ministerio } \\
\text { de } \\
\text { Relaciones } \\
\text { Laborales }\end{array}$ \\
\hline $\begin{array}{c}\text { Control y } \\
\text { Seguimiento }\end{array}$ & \multicolumn{4}{|c|}{$\begin{array}{l}\text { Secretaría Nacional Técnica de Desarrollo de Recursos } \\
\text { Humanos y Remuneraciones del Sector Público, SENRES }\end{array}$} & $\begin{array}{l}\text { Ministerio } \\
\text { de } \\
\text { Relaciones } \\
\text { Laborales }\end{array}$ \\
\hline Aplicación & \multicolumn{5}{|c|}{$\begin{array}{l}\text { Unidades de Administración de Recursos Humanos, UARHs } \\
\text { Unidades de Administración del Talento Humano, UATHs (Desde Octubre } \\
\text { 2010) }\end{array}$} \\
\hline $\begin{array}{l}\text { Ingreso a } \\
\text { Servicio Civil/ } \\
\text { Público }\end{array}$ & \multicolumn{5}{|c|}{$\begin{array}{c}\text { Cumplir con los requerimientos de preparación académica, experiencia y } \\
\text { demás competencias exigibles previstas en el manual genérico de } \\
\text { clasificación de puestos del servicio civil y en los manuales de } \\
\text { clasificación de puestos de cada entidad. Ingreso a un puesto público: } \\
\text { efectuado mediante concurso de merecimientos y oposición, con los } \\
\text { cuales se evalúe la idoneidad de los interesados y se garantice el libre } \\
\text { acceso a los mismos. }\end{array}$} \\
\hline
\end{tabular}

Fuente: elaboración propia.

Tabla 1 B. Institucionalidad y características de ingreso. Fase 2.

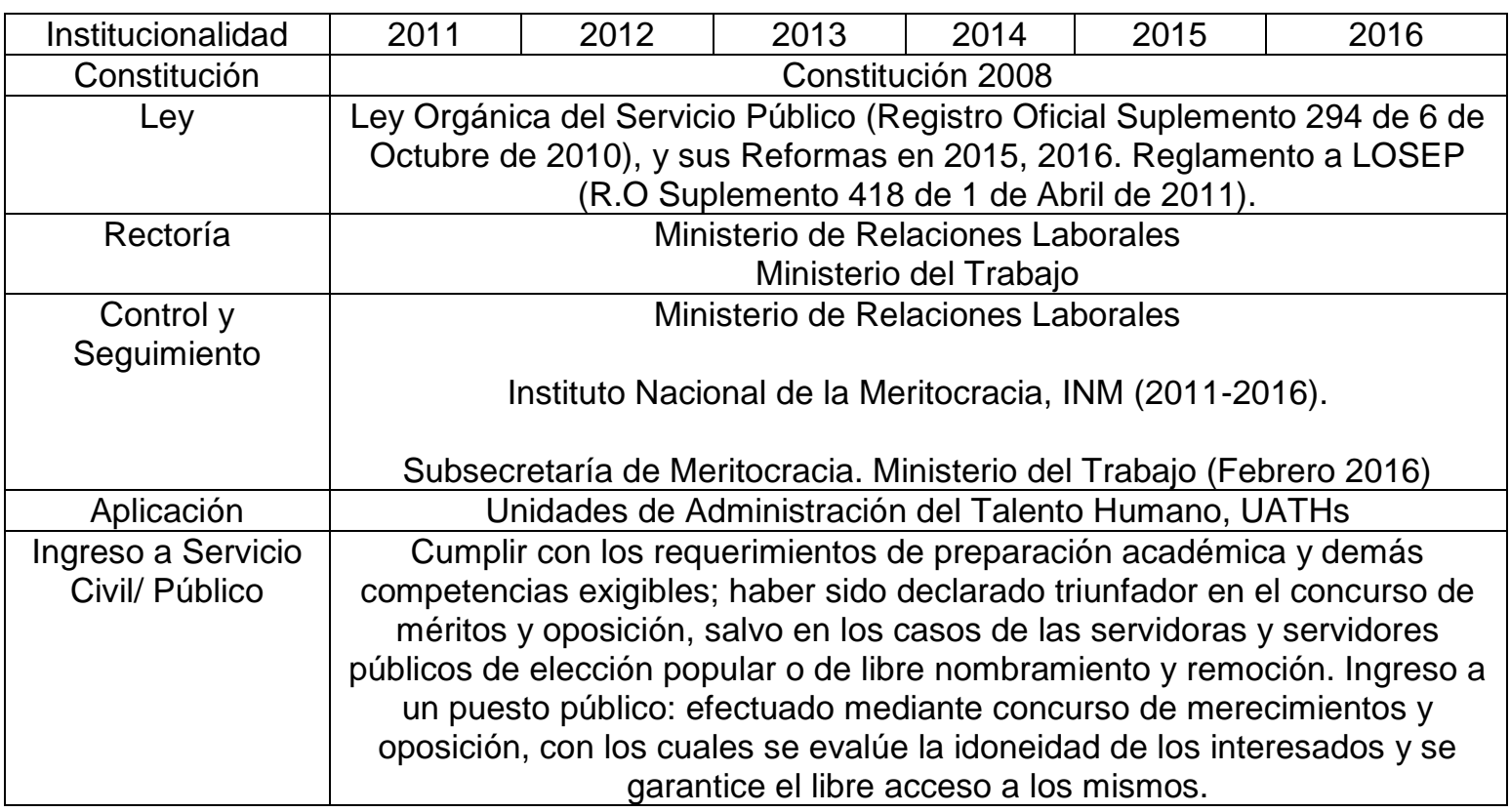

Fuente: elaboración propia. 
Tabla 2 A. Características del sistema meritocrático. Ingreso y carrera. Fase 1.

\begin{tabular}{|c|c|c|c|c|c|}
\hline $\begin{array}{c}\text { Institucionalida } \\
\mathrm{d}\end{array}$ & 2006 & 2007 & 2008 & 2009 & 2010 \\
\hline $\begin{array}{c}\text { Ingreso a } \\
\text { Carrera } \\
\text { Administrativa }\end{array}$ & \multicolumn{5}{|c|}{$\begin{array}{l}\text { Mediante concurso de merecimientos y oposición; demostrar capacidad } \\
\text { mediante el procedimiento de evaluación de desempeño (periodo de prueba); } \\
\text { requisitos del puesto más los del ingreso a servicio civil }\end{array}$} \\
\hline $\begin{array}{l}\text { Proceso de } \\
\text { Selección }\end{array}$ & \multicolumn{5}{|c|}{$\begin{array}{c}\text { Programas de reclutamiento para seleccionar candidatos } \\
\text { Pruebas para el ingreso de los aspirantes a puestos } \\
\text { Concursos de méritos y oposición, bajo las modalidades de comparecencia, sin } \\
\text { comparecencia, abiertos, cerrados y otros; } \\
\text { Nómina de elegibles }\end{array}$} \\
\hline Período de & \multicolumn{5}{|c|}{$\begin{array}{c}\text { Período de prueba de seis meses, evalúa el jefe inmediato. } \\
\text { Se puede solicitar cesación mediante evaluación, aprobada por la unidad de } \\
\text { administración de recursos humanos. }\end{array}$} \\
\hline Evaluación & \multicolumn{5}{|c|}{$\begin{array}{c}\text { Se aplica para el Ingreso, ascenso, cesación y estímulos. } \\
\text { Las evaluaciones a los servidores públicos se realizarán por lo menos una vez } \\
\text { al año. } \\
\text { El servidor evaluado podrá solicitar por escrito la reconsideración. La decisión } \\
\text { sobre el recurso ejercido corresponderá a la autoridad nominadora. Cuando la } \\
\text { evaluación es deficiente será calificado a los tres meses, de no superar será } \\
\text { destituido. }\end{array}$} \\
\hline Ascenso & \multicolumn{5}{|c|}{$\begin{array}{c}\text { Mediante, concurso de merecimientos y oposición en principio de los servidores } \\
\text { opcionados, } \\
\text { Se considera la eficiencia de los servidores medida a través de la evaluación del } \\
\text { desempeño y, complementariamente, los años de servicio. } \\
\text { La unidad de administración de recursos humanos elaborará la correspondiente } \\
\text { nómina de elegibles. }\end{array}$} \\
\hline $\begin{array}{l}\text { Resultados } \\
\text { Mediciones } \\
\text { (BID). Ver Nota }\end{array}$ & \multicolumn{5}{|c|}{$\begin{array}{c}\text { Índice, Gestión del Empleo: } 23 \\
\text { Índice, Gestión del Rendimiento:20 } \\
\text { Índice, Mérito: } 20 \\
\text { Índice de Desarrollo del Servicio Civil: } 15\end{array}$} \\
\hline $\begin{array}{l}\text { Resultado } \\
\text { Seguimiento } \\
\text { MESICIC }\end{array}$ & \multicolumn{5}{|c|}{$\begin{array}{l}\text { Informe Final País, diciembre 2006. Alto nivel de contratación ocasional, no } \\
\text { regulada. Existencia de otros regímenes que no se acogen a la LOSCCA. } \\
\text { Informe Final País, marzo 2010. Alto nivel de contratación ocasional, no } \\
\text { regulada. La SENRES revisa los procesos para otorgar nombramientos. Se } \\
\text { encuentra en elaboración propuesta de reformas a LOSCCA. }\end{array}$} \\
\hline
\end{tabular}

Fuente: elaboración propia a partir de BID (2015); (BID, 2006); MESICIC (2006); MESICIC (2010).

Nota: Resultados recalculados según Longo e lacoviello (2010) para 2004 sobre la base de BID (2006) y hallazgos de los diagnósticos nacionales 2011-13, 2015. Los índices se calculan sobre 100. 
Tabla 2 B. Características del sistema meritocrático. Ingreso y carrera. Fase 2.

\begin{tabular}{|c|c|c|c|c|c|c|}
\hline Institucionalidad & 2011 & & 2013 & 2014 & 2015 & \\
\hline Ingreso a Carrera & \multicolumn{6}{|c|}{$\begin{array}{l}\text { Cumplir con los requisitos exigibles para el desempeño del puesto; } \\
\text { declarado ganador del concurso; posesionarse del cargo }\end{array}$} \\
\hline & \multicolumn{6}{|c|}{$\begin{array}{c}\text { Concursos de méritos y oposición abiertos, parámetros objetivos. Pruebas } \\
\text { de conocimientos, psicológicas, entrevista basada en competencias. } \\
\text { Contratar o nombrar personas con discapacidad o con enfermedades } \\
\text { catastróficas, promoviendo acciones afirmativas para ello, de manera } \\
\text { progresiva y hasta un } 4 \% \text { del total. } \\
\text { Durante la calificación de los concursos de méritos y oposiciones, se } \\
\text { otorgará un puntaje adicional a los aspirantes que demuestren haber sido } \\
\text { acreedores a un reconocimiento dentro del Programa Nacional de } \\
\text { Reconocimientos a la Excelencia Académica. Nota: A la fecha de cierre del } \\
\text { estudio no se encuentra implementado }\end{array}$} \\
\hline Período de Prueba & \multicolumn{6}{|c|}{$\begin{array}{l}\text { Para período de prueba de tres meses, evalúa el jefe inmediato. Para } \\
\text { ascensos periodo de prueba seis meses, sino supera la evaluación regresa } \\
\text { al puesto anterior. Se puede solicitar cesación mediante evaluación, } \\
\text { aprobada por la unidad de administración de recursos humanos. }\end{array}$} \\
\hline Evaluación & \multicolumn{6}{|c|}{$\begin{array}{l}\text { Para ascenso, cesación y estímulos. Las evaluaciones a los servidores } \\
\text { públicos se realizarán una vez al año. Nota: Para efectos del ascenso en la } \\
\text { práctica no se aplica el resultado de la evaluación, ya que los concursos } \\
\text { son abiertos. } \\
\text { El servidor evaluado podrá solicitar por escrito la reconsideración y/o } \\
\text { recalificación, la decisión corresponderá a la autoridad nominadora. La } \\
\text { recalificación realiza un tribunal. Si la evaluación es insuficiente será } \\
\text { destituido de su puesto, previo el respectivo sumario administrativo. Si es } \\
\text { regular será evaluado nuevamente en tres meses. }\end{array}$} \\
\hline Ascenso & \multicolumn{6}{|c|}{$\begin{array}{c}\text { Se realizarán mediante concurso de méritos y oposición, en el que se } \\
\text { evaluará primordialmente la eficiencia de los servidores y, } \\
\text { complementariamente, los años de servicio. Se deberá cumplir con los } \\
\text { requisitos establecidos para el puesto. Nota: la eficiencia en la práctica se } \\
\text { evalúa con los mismos parámetros a todos los postulantes, no se realiza } \\
\text { diferenciación con los resultados del desempeño de los servidores } \\
\text { actuales. }\end{array}$} \\
\hline & \multicolumn{6}{|c|}{$\begin{array}{c}\text { índices al 2011;2015 } \\
\text { Índice, Gestión del Empleo: } 33 ; 53 \\
\text { Índice, Gestión del Rendimiento:20;20 } \\
\text { Índice, Mérito: } 27 ; 53 \\
\text { Índice de Desarrollo del Servicio Civil: } 21 ; 41\end{array}$} \\
\hline & \multicolumn{6}{|c|}{$\begin{array}{l}\text { Informe Final País, marzo 2014. Se recomienda al País la adopción de } \\
\text { sistema de selección abierto de servidores públicos, en todos los casos } \\
\text { Informe Final País, septiembre } 2016 \text {. Tribunal de méritos y oposición y de } \\
\text { apelaciones conformado por servidores de la institución contratante. } \\
\text { Tiempo de convocatoria y difusión se percibe como muy corto favoreciendo } \\
\text { a servidores que ya están en la Entidad. } \\
\text { Se recomienda ejecutar concursos planificados, revisar: porcentaje de } \\
\text { ocasionales, tiempo de difusión de concursos, conformación del tribunal }\end{array}$} \\
\hline
\end{tabular}

Fuente: elaboración propia a partir de BID (2015); (BID, 2006); MESICIC (2006); MESICIC (2010). 
En el ingreso a la carrera, no se consideran a los contratos ocasionales los cuáles son autorizados por la autoridad nominadora, para satisfacer necesidades institucionales, previo el informe de la unidad de administración del talento humano.

Se destaca que en ambas fases, pese a distintos cambios en la institucionalidad una constante es la aplicación y ejecución descentralizada de los procesos para aplicar los sistemas meritocráticos tanto en el ingreso, como en la evaluación durante la carrera. Al respecto Longo (2001) indica la existencia de un consenso que nadie discute cuando se habla de reforma administrativa respecto a la conveniencia de transferir responsabilidades de gestión de recursos humanos desde las instancias especializadas de la administración central a las entidades ejecutoras.

En forma general se evidencia que en la primera evaluación al 2004, el servicio civil ecuatoriano reflejaba debilidades en el sistema de mérito, al 2011 se modificó el ingreso a la administración pública a través de concursos de mérito y oposición de modalidad abierta y al 2015 el sistema de méritos mostró visibles avances, según el análisis y medición realizada por el BID (BID, 2006) (BID, 2015). Al 2016 se generan nuevos cambios en la institucionalidad ya que el Ministerio del Trabajo absorbe al Instituto Nacional de la Meritocracia, en concordancia con un proceso de optimización institucional del sector público; no obstante, los procesos de seguimiento, control de los concursos de méritos y oposición, la recepción de denuncias y las acciones de fortalecimiento de las UATHs se continúan ejecutando a través de la Subsecretaría de Meritocracia, hasta el cierre de este estudio.

Entre las observaciones resultantes de la primera medición del índice de desarrollo de servicio civil al 2004 (BID, 2006), y que se mantienen durante la primera fase de este análisis, se destacan las siguientes:

- No se mantiene actualizada la nómina de los puestos vacantes

- No se publican los concursos en internet y es posible que los candidatos presenten sus hojas de vida directamente a la unidad de personal de cada institución

- El Tribunal de Mérito y Oposición define previamente la clase de concurso (abierto o cerrado) y sus procedimientos

- El Tribunal de Méritos y Oposición evalúa a los candidatos (pruebas, entrevistas y actas), pero no se comprueban directrices y/o fortalecimiento técnico para la aplicación transparente, eficiente y equitativa de las herramientas, pueden contratarse o diseñarse internamente. Si bien se verificó el cumplimiento de formalidades, en la práctica no es demostrable la existencia de un concurso pleno, siendo evidente la 
injerencia política

- No existen procedimientos explícitos sobre pautas para el reclutamiento.

- Los órganos de selección (UARHs, Tribunal) no siempre mantienen criterios de profesionalidad y experiencia técnica

- El procedimiento, en algunos casos es corto y en otros es demasiado burocrático

- Para los servidores de libre remoción no se establece pautas de selección

- Existen contrataciones ocasionales y contratos por servicios profesionales, los cuáles no siguen ningún proceso de concurso

- Las directrices o estándares de evaluación del desempeño esperado no se definen con base a la estrategia y visión organizativa y, los objetivos del proceso no se comunican adecuadamente

- El procedimiento de evaluación del desempeño es aplicado rutinariamente, convirtiéndose en un mero trámite

- No se vincula la evaluación del desempeño a los resultados de la organización ni en términos de desarrollo personal y de carrera. (BID, 2006)

Entre las observaciones que se destacan en la medición al 2011 (BID, 2014) (BID, 2015), se destacan las siguientes:

- Se ocupan vacantes mediante concursos abiertos, públicos y de oposición de méritos

- El tribunal de méritos y oposición realiza el proceso de selección

- EI INM realiza un informe previo a la declaratoria de ganador, tenía la facultad de vigilar y controlar el desarrollo del proceso y de emitir su anulación (desierto) en caso de anomalías

- El mecanismo más usado para cubrir los puestos seguía siendo por contrato ocasional para los cuales no se siguen necesariamente los requerimientos de los manuales o se aplican pruebas de algún tipo

- En la evaluación no se observa que los objetivos, metas e indicadores reflejen las actividades ejecutadas, no existe la comunicación adecuada de los estándares, indicadores, alcance y consecuencias de la evaluación; existen quejas de que se modifican metas para favorecer o perjudicar a los servidores y por la tanto no se releva el mérito. (BID, 2015).

Entre las observaciones que se destacan en la medición al 2015 (BID, 2015), se destacan las siguientes: 
- Creció la cantidad de vinculaciones a través de concursos de méritos y oposición

- El INM realiza seguimiento, control y asistencia técnica

- La norma regula en detalle el proceso para selección de servidores de carrera y, se regula las bases, convocatoria, pruebas, entrevista, puntaje y criterios de competencia a evaluar, disposiciones orientadas a garantizar las acciones afirmativas, la transparencia del proceso, y la posibilidad de presentar apelaciones

- Se modificaron los pesos de los parámetros de calificación

- Mejoró la confiabilidad de las pruebas, existe la opción de acceder al Banco de preguntas del INM

- Se redujo mediante normativa la duración del proceso (de 134 a 38 días)

- Se conformó el banco de elegibles (vigencia dos años)

- Se diseñó, aunque de manera opcional, un programa específico para los directivos públicos y se mejoró -en relación al anterior periodo- la informatización; el proceso no es normado (BID, 2015)

Durante el periodo 2011-2016, aunque existe aún la modalidad de contratación ocasional, este proceso es controlado, conforme la normativa por el ente rector, dentro del porcentaje determinado en la LOSEP; no obstante, siguen existiendo excepciones autorizadas, como por ejemplo aquellas relacionadas con contrataciones en instituciones nuevas, proyectos de inversión, además de las de puestos enmarcados en el grupo del jerárquico superior, y también se realizan contrataciones bajo la modalidad de servicios profesionales.

Respecto a los informes del MESICIC, en el Informe final de 15 de diciembre de 2006, aprobado en la décima reunión del comité de expertos, sobre la implementación de las disposiciones de la convención seleccionadas para ser analizadas en la segunda ronda, entre las cuáles estaba el referido a los sistemas para la contratación de funcionarios públicos que aseguren la publicidad, equidad y eficiencia, y sobre el seguimiento de las recomendaciones formuladas en la primera ronda, se destaca que el marco constitucional y legal referido al sistema de ingreso es pertinente para la promoción de los propósitos de la convención interamericana contra la corrupción, pero se tienen las siguientes observaciones, por parte del mecanismo (MESICIC; 2006):

- Existe preocupación respecto a las contrataciones por servicios ocasionales, ya que aunque están limitadas al año fiscal, la excepción posibilita la extensión por las necesidades del servicio, lo que podría dar lugar a renovaciones continuas evitando el 
concurso de méritos y oposición .

- Se perciben algunos vacíos e inconsistencias en aquellos sistemas que están excluidos de la LOSCCA y que se rigen por sus propias leyes

En el Informe final de 25 de marzo de 2010, aprobado en la décima sexta reunión del comité de expertos, sobre el seguimiento de las recomendaciones de rondas anteriores, en lo respectivo a contratación de servidores, se indica que la gestión de la SENRES se focaliza en la revisión de nombramientos en todo el sector público y se trabaja en propuesta de la reforma a la LOSCCA, para fortalecer los sistemas para la contratación de los funcionarios en general del servicio público, y adaptándola a la nueva Constitución. (MESICIC, 2010)

En el Informe final de 19 de marzo de 2014, aprobado en la vigésimo tercera reunión del comité de expertos, sobre el seguimiento de las recomendaciones de rondas anteriores, en lo respectivo a contratación de servidores, se recomienda la adopción del subsistema de selección de personal como abierto en todos los casos, para transparentar el proceso de contratación de servidores públicos de conformidad con la LOSEP. (MESICIC, 2014) En el Informe final de 15 de septiembre de 2016, aprobado en la vigésimo séptima reunión del comité de expertos, sobre el seguimiento a la implementación de recomendaciones de segunda ronda y de las recomendaciones de rondas anteriores, en lo respectivo a contratación de servidores, indica, que:

- Se reconoce que los nuevos desarrollos normativos como aspectos positivos que se han dado en el sentido de establecer un sistema más armónico para el ingreso por méritos de los servidores a la carrera administrativa de las instituciones y organismos del sector público. Sin embargo, se recomienda analizar los porcentajes de utilización de los contratos ocasionales y ejecutar los concursos planificados, para disminución de los nombramientos provisionales.

- Los nombramientos provisionales se utilizan, entre otros, para ocupar puestos vacantes desde la planificación del concurso hasta la declaratoria de ganador.

- La conformación del Tribunal de méritos y oposición encargado de la declaratoria de ganador y el de las apelaciones consiste únicamente de servidores de la institución contratante $y$, aunque no son las mismas personas las que conforman los tribunales esto se percibe como una debilidad al no considerarse a esta conformación como de segunda parte. Se recomienda revisar su conformación.

- Se percibe como muy corto el plazo entre la publicación y difusión y la postulación lo 
que podría dar ventajas a los candidatos internos respecto a la preparación de su postulación MESICIC. Se recomienda ampliar el plazo.

- El comité toma nota del fortalecimiento de los roles del MDT como autoridad rectora de los sistemas de selección del personal general y de la norma específica para los procesos de concursos de oposición y mérito (MESICIC, 2016)

\section{Evolución y mejora del sistema.}

En el estudio, se analizó por fases la política pública de meritocracia, enfocada en el ingreso y la evaluación del desempeño, contrastando la normativa, implementación y alcance de resultados con la existencia y cumplimiento de los elementos fundamentales que caracterizan un sistema meritocrático.

Los elementos considerados para el ingreso fueron: concurso de mérito como mecanismo de ingreso; concursos abiertos y de amplia difusión; concursos basados en pruebas técnicas de selección (que incluyan competencias); publicidad, transparencia en la gestión de los procesos; idoneidad e imparcialidad de los cuerpos técnicos encargados de la selección; fiabilidad de las herramientas que se instrumentan para la selección; equidad y eficiencia.

En cuanto a la evaluación, se consideró si los siguientes elementos eran aplicados para el efecto: evaluación institucional; evaluación para altos funcionarios públicos; evaluación individual.

En general respecto al sistema, se investiga si ofrece el grado de protección efectiva frente a la arbitrariedad, la captura política o clientelista y las diferentes modalidades de búsqueda de rentas por parte de grupos o sectores interesados.

La tabulación del análisis se muestra que en el periodo de estudio, de 13 elementos considerados como fundamentales para un sistema meritocrático, en la fase 1 respecto al ingreso, se cumple con apenas uno, que corresponde al relacionado con la existencia de concurso de méritos; para la evaluación se cumple con uno, que se refiere a la existencia de la evaluación individual de desempeño; por lo que en esta fase no se asegura la protección efectiva contra la arbitrariedad y clientelar con el sistema implementado.

Al final de la fase 2, respecto al ingreso se cumple con siete de nueve elementos, quedando aún por cumplir lo relacionado con la idoneidad e imparcialidad de los cuerpos técnicos y la eficiencia en el proceso de ingreso; para la evaluación se cumple con uno, que se refiere a la existencia de la evaluación individual de desempeño, por lo que en 
esta fase tampoco se asegura, de forma plena, la protección efectiva contra la arbitrariedad y clientelar con el sistema implementado aunque se observan importantes logros.

De forma comparada diacrónica, se observa la evolución en mejora en cuanto al sistema meritocrático, ya que se cumplen con 5 de 9 elementos del sistema meritocrático para el ingreso, por lo que, el avance es significativo; no así, en lo referido a evaluación de desempeño, donde básicamente no se registran avances.

Tabla 4. Cumplimiento de elementos fundamentales.

\begin{tabular}{|c|c|c|c|c|c|c|c|c|c|}
\hline Elementos / Aspectos & $\mathrm{N}$ & I & $\mathrm{R}$ & $\begin{array}{l}\text { Fase } 1 \\
(2006- \\
2010)\end{array}$ & $\mathrm{N}$ & $\mathrm{I}$ & $\mathrm{R}$ & $\begin{array}{l}\text { Fase } 2 \\
(2011- \\
2016)\end{array}$ & $\begin{array}{l}\text { Evolución } \\
\text { / Mejora }\end{array}$ \\
\hline Ingreso & & & & $2 / 9$ & & & & $7 / 9$ & $5 / 9$ \\
\hline Concurso de mérito & 1 & 1 & 0 & 1 & 1 & 1 & 1 & 1 & 0 \\
\hline Concurso abierto & 1 & 0 & 0 & 0 & 1 & 1 & 1 & 1 & 1 \\
\hline Pruebas de Selección & 1 & 1 & 0 & 1 & 1 & 1 & 1 & 1 & 1 \\
\hline Publicidad procesos & 1 & 0 & 0 & 0 & 1 & 1 & 1 & 1 & 1 \\
\hline Transparencia Procesos & 1 & 0 & 0 & 0 & 1 & 1 & 1 & 1 & 1 \\
\hline $\begin{array}{l}\text { Idoneidad e imparcialidad } \\
\text { cuerpos técnicos }\end{array}$ & 1 & 0 & 0 & 0 & 1 & 0 & 0 & 0 & 0 \\
\hline Fiabilidad de herramientas & 0 & 0 & 0 & 0 & 1 & 1 & 0 & 1 & 1 \\
\hline Equidad & 0 & 0 & 0 & 0 & 1 & 1 & 0 & 1 & 1 \\
\hline Eficiencia & 0 & 0 & 0 & 0 & 1 & 0 & 0 & 0 & 0 \\
\hline Evaluación & & & & $1 / 3$ & & & & $1 / 3$ & $0 / 3$ \\
\hline Evaluación Institucional & 0 & 0 & 0 & 0 & 0 & 0 & 0 & 0 & 0 \\
\hline Evaluación Individual & 1 & 1 & 0 & 1 & 1 & 1 & 0 & 1 & 0 \\
\hline $\begin{array}{l}\text { Evaluación Altos } \\
\text { Funcionarios }\end{array}$ & 0 & 0 & 0 & 0 & 0 & 0 & 0 & 0 & 0 \\
\hline \multicolumn{10}{|l|}{ Sistema } \\
\hline $\begin{array}{l}\text { Protección Efectiva contra } \\
\text { arbitrariedad y clientelar/ } \\
\text { Todo el Sistema }\end{array}$ & 1 & 0 & 0 & 0 & 1 & 0 & 0 & 0 & 0 \\
\hline
\end{tabular}

Fuente: elaboración propia.

\section{CONCLUSIONES}

Ecuador evidencia un avance importante en el ámbito normativo, de estructuras, organizaciones, procesos y acciones en cuanto al desarrollo del sistema meritocrático de la administración central, desde los aspectos de selección para el ingreso a la carrera administrativa. La administración pública ha organizado el sistema, desarrollado instrumentos e implementado procesos a fin de responder a las demandas de un sistema democrático, para el servicio civil basado en méritos; para ello, se ha requerido a lo largo de estos años el incremento de las capacidades, infraestructura tecnológica, avances de interoperabilidad y varias reformas normativas. 
En relación a las diferentes reformas identificadas en este periodo, es necesario destacar que si bien estas pueden contribuir al ordenamiento 0 fortalecimiento de la institucionalidad, así como a su adecuado alineamiento al nuevo marco constitucional en el mediano plazo, sin embargo, en corto plazo estos ajustes ocasionan que los procesos e instrumentos no alcancen a desarrollarse de forma eficiente, ya que por motivos de cambios estructurales y organizacionales que se generan, estos deben también ser ajustados, lo que a su vez conlleva a realizar ajustes en los trámites y a que las percepciones de la ciudadanía sean desfavorables ante los constantes cambios no solo en el uso de las herramientas, sino también en la calificación y requisitos de postulaciones. Al respecto, resulta necesario para futuro -previo a cualquier reformaenfocarse en el ámbito institucional, identificando elementos específicos de desempeño, que permitan definir la pertinencia y priorizar las reformas normativas $u$ organizacionales; así como, valorar la efectividad de los resultados de las acciones.

En un análisis macro considerando los diferentes aspectos de institucionalidad para la meritocracia, demostrados en la normativa dispuesta, instituciones rectoras, de seguimiento y control y ejecutoras se verifica la existencia de coherencia en las acciones, se puede observar además que su desarrollo acompaña la tendencia a la mejora en el índice de desarrollo del servicio civil, aunque este siga siendo por debajo de lo óptimo. No obstante, mantener esta tendencia dependerá de que la institucionalidad recientemente modificada concluya su fortalecimiento, y se sostengan las acciones y se desarrollen además de los elementos fundamentales, las buenas prácticas en cuanto a evaluación del desempeño.

Teniendo en consideración las particularidades locales, así como la necesidad imperante de obtener eficiencia en los procesos, debido a la austeridad fiscal requerida, se identifica que es requerido implementar, conforme se recomienda en el último informe de MESICIC (2016), la propuesta de centralizar los concursos desde MDT pero por escalas, progresivo y de forma transversal, a fin que se realicen concursos generales por grupo ocupacional favoreciendo la eficiencia por economía de escala, la equidad al conformarse un solo banco de elegibles para el servicio público y por grupo ocupacional, homologando las pruebas y/o las técnicas en su aplicación y, oportunidad en la selección.

Finalmente, se resalta en lo cuantitativo que del análisis diacrónico se determinaron avances en cinco (5) elementos de meritocracia en el sistema Ecuador -en los que se cumplen al menos con 2 de los 3 aspectos- en la selección: concursos realizados por 
mérito, modalidad abierta, oposición que incluye aplicación de pruebas, publicidad, transparencia, finalidad en la herramientas y equidad en la calificación.

\section{REFERENCIAS BIBLIOGRÁFICAS}

Asamblea Nacional Constituyente (1998). Constitución de la República del Ecuador. Aprobada el 5 de junio del 1998.

Asamblea Nacional (2007). Constitución de la República del Ecuador. Codificada con modificaciones de Referendum y Consulta Popular del 7 de mayo del 2011.

Registro Oficial No. 44920 de octubre de 2008, Quito, Ecuador.

Asamblea Nacional (2010). Ley Orgánica del Servicio Público. Registro Oficial Suplemento No. 294 de 6 de octubre de 2010, Quito, Ecuador.

BID (2001). Instituciones, reforma del estado y desarrollo: de la teoría a la práctica. BID, División de Estado, Gobernabilidad y Sociedad Civil. Escuela de Cooperación Internacional al Desarrollo "Raúl Prebisch": Nuevas estrategias en cooperación y desarrollo. Hacia una agenda comprensiva para el desarrollo. Universidad Internacional Menéndez y Pelayo, Santander.

BID (2006). Informe sobre la situación del servicio civil en América Latina. Washington, DC: BID. Editor: Koldo Echeverría.

BID.División de Capacidad Institucional del Estado (2014). Al servicio del ciudadano: una década de reformas del servicio civil en América Latina (2004-13). (M. L. Juan Carlos Cortázar Velarde, Ed.) Washington.

BID (2015). Diagnóstico Institucional del servicio civil, República del Ecuador. Washington, DC: BID. Responsable: Luciano Strazza.

Borja, R. (2012). Enciclopedia de la Política, tomo II: H-Z (4a. Ed. ed.). México, México: Fondo de Cultura Económica. 
CLAD (2003). Carta Iberoamericana de la Función Pública. (Documentos Clave: Estado, Administración Pública y Sociedad; № 9). Caracas: CLAD.

Congreso Nacional (2005). Ley Orgánica de Servicio Civil y Carrera Administrativa. Registro Oficial Suplemento No. 16 de 12 de mayo de 2005, Quito, Ecuador.

lacoviello, M. (2014). Diagnóstico Institucional del Sevicio Civil en América Latina: Ecuador. BID.

lacoviello, M. y Chudnovsky, M. (2015). La importancia del servicio civil en el desarrollo de las capacidades estatales de América Latina. Corporación Andina de Fomento.

Jara, I. (2015). Cómo deben ser consideradas las características y limitaciones locales en las teorías de construcción de las políticas públicas. Estudio de las políticas de capacitación en el Ecuador: 1978-2011. Tesis Doctoral. Universidad Andina Simón Bolívar, Quito, Ecuador.

Longo, F. (2004). Mérito y flexibilidad: la gestión de las personas en las organizaciones del sector público. Barcelona: Paidós.

Longo, F. (2006). Marco analítico para el diagnóstico institucional de Sistemas de Servicio Civil. En: BID, Informe sobre la situación del servicio civil en América Latina. Washington, DC: BID.

Longo, F. y M. lacoviello. (2010). Evaluación del grado de implantación de la Carta Iberoamericana de la Función Pública en los países de América Latina. Documento preparado para la Reunión de Ministros de Función Pública del Centro Latinoamericano para el Desarrollo (CLAD), Buenos Aires, 1 y 2 de julio.

MESICIC (2006). Informe Final. República del Ecuador. Décima Reunión del Comité de Expertos Del 11 al 16 de diciembre de 2006 Washington, DC. 
MESICIC (2010). Informe Final. República del Ecuador. Decimosexta Reunión del Comité de Expertos Del 22 al 26 de marzo de 2010 Washington, DC.

MESICIC (2014). Informe Final. República del Ecuador. Vigésima Tercera Reunión del Comité de Expertos Del 17 al 21de marzo de 2014 Washington, DC.

MESICIC (2016). Informe Final. República del Ecuador. Vigésimo Séptima Reunión del Comité de Expertos Del 12 al 15 de septiembre de 2016 Washington, DC.

OEA (1996). Convención Interamericana Contra la Corrupción. (S. G. OEA, Ed.) Caracas, Venezuela.

OEA (2011). Departamento de Cooperación Jurídica. (D. d.-S. Jurídicos, Productor, \& OEA) Recuperado el 22 de Enero de 2017, de OEA: http://www.oas.org/juridico/spanish/mesicic_intro_sp.htm

Real Academia Española (2014). Diccionario de la Lengua española. (R. A. Española, Productor) Recuperado el 20 de 01 de 2017, de Real Academia Española: http://www.rae.es/obras-academicas/diccionarios/diccionario-de-la-lenguaespanola

\section{Licencia Creative Commons}

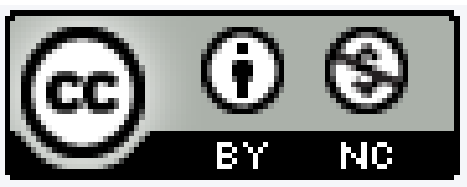

Revista Científica ECOCIENCIA está bajo una Licencia Creative Commons Atribución-NoComercial 4.0 Internacional. 